\section{A case of delayed bleeding after endoscopic submucosal dissection for completely circum- ferential esophageal cancer}

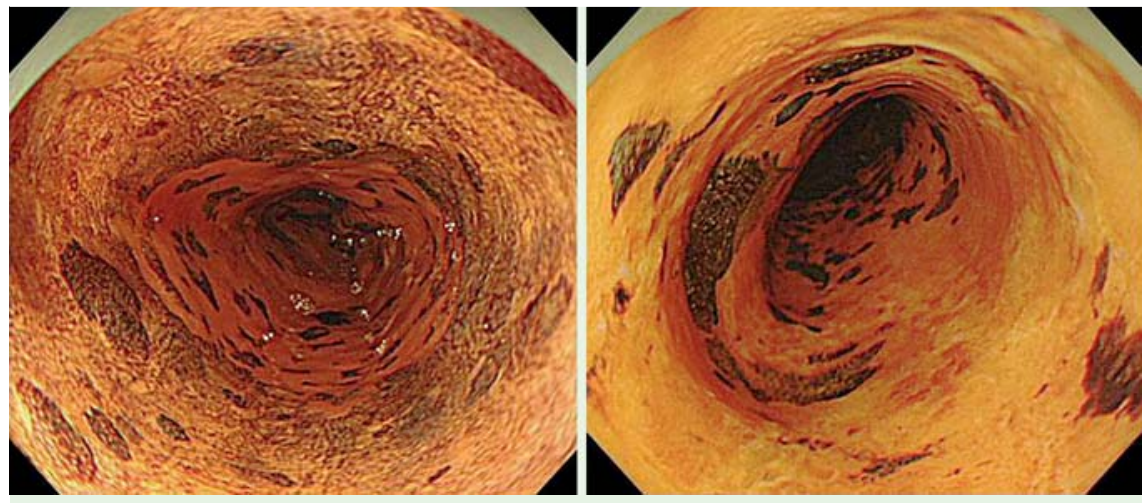

Fig. 1 Endoscopic views with iodine staining of a superficial cancer that involves the entire circumference of the esophageal lumen in an 82-year-old man.
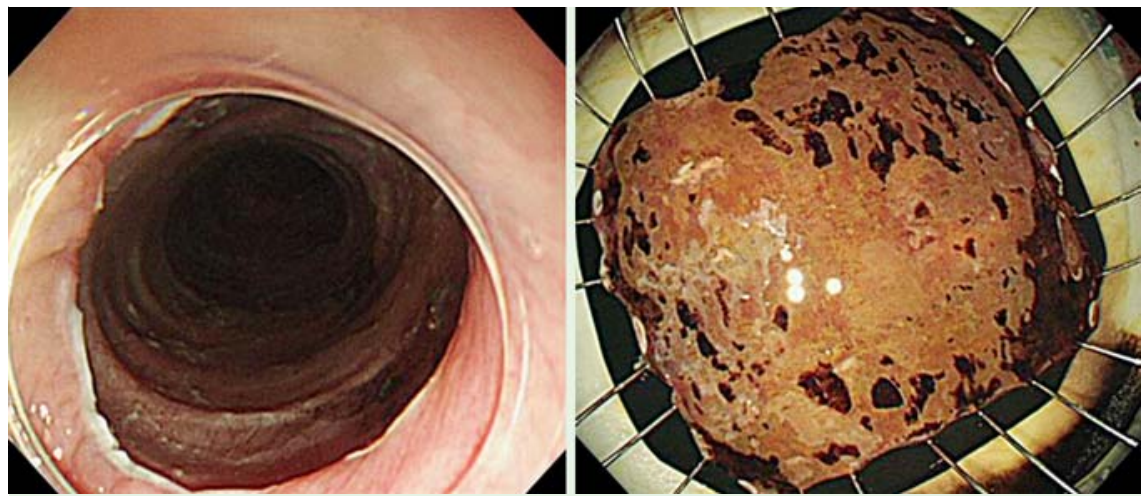

Fig. 2 Circumferential endoscopic submucosal dissection is performed without any complications.

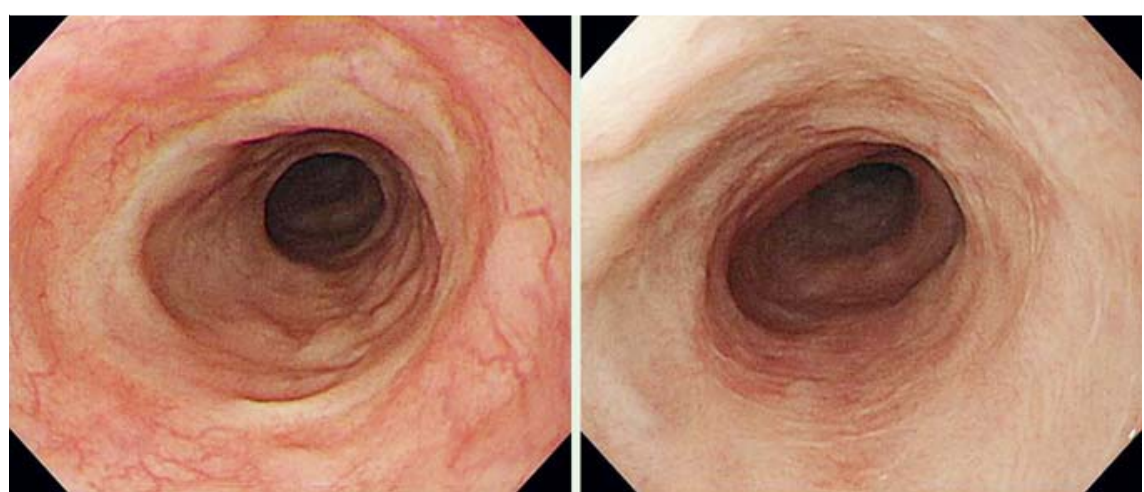

Fig. 3 At 1 year after endoscopic submucosal dissection, the ulcer has healed completely without stricture.

Endoscopic submucosal dissection (ESD) for early esophageal cancer is being performed more frequently. Perforation and stricture are well-known complications of esophageal ESD, but delayed bleeding is very rare. This is the first report of delayed bleeding after esophageal ESD.
An 82-year-old man was admitted to our hospital to undergo esophageal ESD for a superficial esophageal cancer $60 \mathrm{~mm}$ in diameter that involved the entire circumference of the esophageal lumen ( $\bullet$ Fig. 1). En bloc resection of the tumor was performed successfully without any complications ( $\bullet$ Fig.2). To prevent postESD stricture, triamcinolone acetonide was injected into the remaining submucosa immediately after ESD.

The following morning, the patient vomited a large volume of blood, and an emergency endoscopy was performed. After numerous clots had been removed with grasping forceps, the bleeding was stopped with hemostatic forceps ( $\bullet$ Video $\mathbf{1}$ ). Fortunately, endoscopic balloon dilation was not required after one local injection and the systemic administration of prednisolone. The patient was able to ingest all foods without any symptoms at 1 year after ESD (৫ Fig. 3).

Bleeding after esophageal ESD is very rare. From March 2007 to March 2015, the rate, including this case, was $0.19 \%(1 / 529)$ in our experience. Tsujii et al. reported no cases of post-ESD bleeding in 368 patients [1]. This absence of post-ESD bleeding is unexplained but may be related to the lower level of exposure to gastric acid after esophageal ESD than after gastric ESD. In this patient, hypertension (systolic blood pressure $>190 \mathrm{mmHg}$ ) after ESD may also have played a role.

In patients with post-ESD bleeding, clips are typically effective for hemostasis. However, in circumferential ESD for esophageal cancer, hemostatic forceps should be used because such lesions are associated with a high risk for post-ESD stricture, which may require endoscopic balloon dilation.

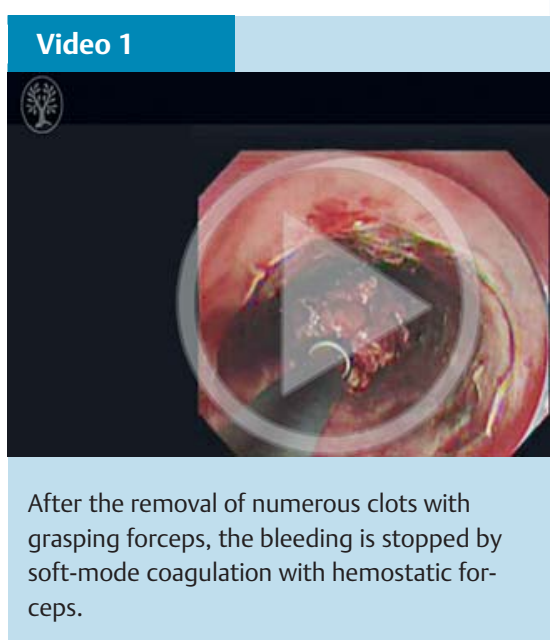

Endoscopy_UCTN_Code_CPL_1AJ_2AD

Competing interests: None 
Hideyuki Chiba', Keiichi Ashikari', Akihiro Takahashi', Toru Goto', Ken Ohata ${ }^{2}$, Nobuyuki Matsuhashi², Atsushi Nakajima ${ }^{3}$

${ }^{1}$ Department of Gastroenterology, Omori Red Cross Hospital, Tokyo, Japan

2 Department of Gastroenterology, NTT Medical Center Tokyo, Tokyo, Japan

${ }^{3}$ Department of Gastroenterology, Yokohama City University, Kanagawa, Japan

\section{Reference}

1 Tsujii Y, Nishida T, Nishiyama $O$ et al. Clinical outcomes of endoscopic submucosal dissection for superficial esophageal neoplasms: a multicenter retrospective cohort study. Endoscopy 2015; 47. Epub 2015 March 31. DOI: $10.1055 / \mathrm{s}-0034-1391844$

\section{Bibliography}

Dol http://dx.doi.org/

10.1055/s-0034-1392429

Endoscopy 2015; 47: E385-E386

(c) Georg Thieme Verlag KG

Stuttgart · New York

ISSN 0013-726X

Corresponding author Hideyuki Chiba, MD, PhD

Omori Red Cross Hospital

Department of Gastroenterology

4-30-1, Chuo

Ota-Ku

Tokyo, 143-8527

Japan

Fax: +81-3-3448-6541

h.chiba04@gmail.com 